\title{
Disfunción en el trastorno por déficit de atención/ hiperactividad: evaluación y respuesta al tratamiento
}

\author{
Alberto Fernández-Jaén, Daniel Martín Fernández-Mayoralas, Ana L. Fernández-Perrone, Beatriz Calleja-Pérez, \\ Jacobo Albert, Sara López-Martín, Sonia López-Arribas
}

\begin{abstract}
Resumen. El trastorno por déficit de atención/hiperactividad (TDAH) es un trastorno heterogéneo y complejo sintomáticamente. Su sintomatología cardinal, la presencia de problemas disejecutivos, la desregulación emocional de muchos de ellos y la propia comorbilidad, entre otros, condicionarán su expresión clínica y la disfunción. La tipificación del TDAH como 'trastorno' requiere una evaluación precisa del término 'disfunción' o 'repercusión'. Los avances en la tipificación y cuantificación de la sintomatología característica del TDAH deberían trasladarse a la medición y objetivación de la disfunción. La estimación de la disfunción como una simple interferencia, por clara que sea, podría llevar a una sobreestimación del diagnóstico de este trastorno. Del mismo modo que es ineludible su estimación para el diagnóstico, es igualmente necesaria para la correcta evaluación de la eficacia de las intervenciones terapéuticas, especialmente a medio y largo plazo. Son necesarios estudios adicionales en este sentido para valorar la eficacia de los tratamientos, sean farmacológicos o no, en diferentes dominios (relación social, aprendizaje, autoestima, calidad de vida, siniestralidad...).
\end{abstract}

Palabras clave. Déficit de atención. Disfunción. DSM-5. Repercusión. TDAH. WFIRS.

\section{Introducción}

El trastorno por déficit de atención/hiperactividad (TDAH) es uno de los trastornos del neurodesarrollo más prevalentes en la población infantojuvenil. Su caracterización clínica básica se sustenta en la presencia de problemas atencionales, hiperactividad e impulsividad; sin embargo, estos síntomas cardinales no siempre están presentes con la misma intensidad e interferencia funcional. De hecho, en la mayoría de los casos, el TDAH se manifiesta sin graves problemas en el apartado hiperactividad-impulsividad. Un reciente estudio metaanalítico ha demostrado una mayor prevalencia del subtipo inatento frente a cualquier otra presentación del TDAH, a pesar de que los pacientes con inatención e hiperactividad son remitidos con mayor frecuencia para valoración diagnóstica y terapéutica [1].

Por otro lado, los síntomas recogidos en las clasificaciones más actuales [2,3] no siempre explican numerosos síntomas que caracterizan a muchos de estos pacientes. La presencia de déficit en el funcionamiento ejecutivo o en la aversión al refuerzo demorado puede explicar otros síntomas no recogidos en las clasificaciones previamente señaladas [4-6]. La flexibilidad cognitiva, la activación, la organización, la planificación o el sentido del tiempo, la monitorización y la memoria de trabajo, cuando están afectadas en el paciente con TDAH, pueden interferir de forma significativa en su aprendizaje social 0 académico.

La desregulación emocional constituye otro aspecto fundamental en el TDAH, aunque tampoco sea uno de los síntomas diagnósticos del TDAH. Los pacientes pueden tener problemas en todas las habilidades básicas que constituyen la competencia social: el reconocimiento, la regulación y la expresión de las emociones $[7,8]$. La desregulación emocional se vincula frecuentemente a la gravedad del TDAH y sus comorbilidades, y, en sí misma, tiene un impacto significativo en el funcionamiento diario del paciente $[7,9]$.

Los factores ambientales, familiares y escolares serán igualmente relevantes en el componente disfuncional del TDAH. La presencia de pobres relaciones parentales, estilos educativos inapropiados o antecedentes de psicopatología en los padres se asocian con un riesgo aumentado para la presencia de TDAH, su gravedad y respuesta a las medidas terapéuticas [10-13]. En el mismo sentido, la formación sobre aspectos básicos del TDAH se ha mostrado necesaria para el colectivo docente $[14,15]$. Esta formación es necesaria para el manejo terapéutico de niños y adolescentes con TDAH. Intervenciones inclusivas pueden atenuar la sintomatología y disminuir el impacto del TDAH [16], así como algunas de sus comorbilidades [17].
Centro CADE (A. Fernández-Jaén, D. Martín Fernández-Mayoralas, A.L. Fernández-Perrone, S. LópezArribas). Unidad de Neurología Infantil; Hospital Universitario Quirón Madrid (A. Fernández-Jaén, D. Martín Fernández-Mayoralas, A.L. Fernández-Perrone). Atención Primaria de Pediatría; Centro de Salud Doctor Cirajas (B. CallejaPérez). Facultad de Psicología; Universidad Autónoma de Madrid (J. Albert). Facultad de Ciencias de la Salud; Universidad Rey Juan Carlos; Centro Neuromottiva (S. López-Martín). Unidad de Psiquiatría Infanto-Juvenil; Hospital Gómez Ulla (S. López-Arribas). Madrid, España.

\section{Correspondencia:}

Dr. Alberto Fernández Jaén. Hospital Universitario Quirón Madrid. Diego de Velázquez, 1. E-28223 Pozuelo de Alarcón (Madrid).

E-mail:

aferjaen@telefonica.net

Declaración de intereses: Los autores manifiestan la inexistencia de conflictos de interés en relación con este artículo.

Aceptado tras revisión externa: 22.01.16.

Cómo citar este artículo:

Fernández-Jaén A, Martín FernándezMayoralas D, Fernández-Perrone AL, Calleja-Pérez B, Albert J, LópezMartín S, et al. Disfunción en el trastorno por déficit de atención/ hiperactividad: evaluación y respuesta al tratamiento. Rev Neurol 2016; 62 (Supl 1): S79-84.

(c) 2016 Revista de Neurología 


\section{Disfunción en el diagnóstico del TDAH}

Las guías nacionales e internacionales para el diagnóstico del TDAH [18-21], apoyándose en las clasificaciones más actuales de la salud mental-Manual diagnóstico y estadístico de los trastornos mentales, cuarta edición (DSM-5) y Clasificación Internacional de Enfermedades, décima revisión (CIE-10) [2,3]-, recomiendan la recogida de los síntomas cardinales del TDAH y la evaluación de la repercusión que éstos generan.

La conceptualización del TDAH tal y como lo entendemos en este momento es realmente novedosa. Debemos recordar que tanto las clasificaciones americanas como las europeas (DSM y CIE) nacen con el propósito de clasificar diferentes procesos, y evolutivamente han sufrido numerosas modificaciones [22]. Desde la descripción de la 'reacción hipercinética de la infancia' del DSM-II al 'trastorno por déficit de atención/hiperactividad' del DSM-5, las modificaciones han afectado predominantemente a los síntomas que lo caracterizan, el número de síntomas que son necesarios para su diagnóstico o la propia subtipificación dentro del diagnóstico del TDAH [23]. Incluso su traducción a nuestro idioma genera confusión, y se ha visto cómo el 'trastorno por déficit de atención/hiperactividad' se sustituye por 'trastorno por déficit de atención con hiperactividad' [24].

Sin embargo, el apartado disfuncional, como criterio diagnóstico, parece mostrarse incomprensiblemente estable en los últimos años y clasificaciones. Es más, en las últimas clasificaciones, la disfunción del TDAH ha perdido peso en el diagnóstico; en las clasificaciones DSM-IV y DSM-IV-TR [25,26], en el apartado $C$ se señalaba que la 'disfunción de los síntomas del TDAH debía estar presente en dos o más entornos', y en el apartado D se describía cómo debía existir 'una clara evidencia de disfunción clínicamente significativa en el funcionamiento social, académico u ocupacional. Es decir, no sólo los síntomas debían estar presentes en dos o más entornos, sino que debían generar una disfunción en los entornos señalados, y la disfunción debía ser realmente significativa. De forma similar, la todavía no actualizada CIE-10 requería para el diagnóstico del 'trastorno de la actividad y de la atención' síntomas de inatención, hiperactividad e impulsividad, que provocaran una clara disfunción al menos en dos ambientes. En contrapartida, el DSM-5 [2] atenúa sorprendentemente el apartado disfuncional; se precisa que los síntomas estén presentes en dos o más ambientes, no así la disfunción; debe haber una clara evidencia de que los síntomas interfieren con o reducen la calidad del funcionamiento social, académico u ocupacional. Críticamente, cabe pensar que una menor capacidad atencional o un menor autocontrol siempre o frecuentemente reducirán estas calidades. Por otro lado, se distingue la gravedad del trastorno no sólo por el deterioro funcional, sino también por el número de síntomas que cumple el paciente. En contrapartida, siguen sin reflejarse otros aspectos funcionales aparentemente relevantes, como la autoestima $[27,28]$, la calidad de vida $[29,30]$ o la siniestralidad [31,32], entre otros.

La sintomatología o el número de síntomas que describen el TDAH tienen una relación significativa, aunque leve, con la disfunción que generan [3335], es decir, una mayor intensidad sintomática o la presencia de más síntomas no siempre se vincula a una mayor repercusión. Esto contrasta con la clasificación de gravedad del TDAH según el DSM-5. Por este motivo, debemos insistir en la necesidad de ponderar cautelosamente la disfunción que generan los síntomas del TDAH. Los estudios en este sentido son escasos, y los instrumentos que se utilizan, muchas veces globales y no multidimensionales, generalmente inespecíficos, escasamente fiables y poco validados [36]. De hecho, es frecuente que la disfunción como tal no se valore o mida adecuada y constantemente en la evaluación de los trastornos del neurodesarrollo [36].

La ausencia de estimación de la disfunción lleva a la sobreestimación del TDAH hasta en un $40 \%$ [1]. En este mismo sentido, es interesante observar cómo aproximadamente la mitad de los estudios de prevalencia de TDAH en nuestro país no han estimado -o al menos reflejado en sus estudios- el criterio D, a pesar de haber 'empleado' los criterios diagnósticos del DSM-III-R o del DSM-IV [37].

\section{Interferencia de la comorbilidad}

Es ampliamente reconocida la elevada comorbilidad que presentan los pacientes con TDAH. Numerosos estudios señalan la elevada frecuencia en pacientes con TDAH de problemas asociados, como los trastornos específicos del aprendizaje, el trastorno oposicionista desafiante, los trastornos por ansiedad o del ánimo, el trastorno de Tourette o los trastornos de la conducta [38-40]. Por este motivo, entre otros, la mejora en los síntomas cardinales no siempre es la única meta terapéutica en el TDAH [40].

Si en más del $60 \%$ de los casos los pacientes tienen al menos una comorbilidad tipificable según el DSM $[38,40]$, la valoración del componente disfuncional se muestra más compleja, y se separa eviden- 
temente de la gravedad sintomática o del número de síntomas propios del TDAH que presenta el paciente. Numerosos estudios han señalado a los trastornos específicos del aprendizaje y al trastorno oposicionista-desafiante como los más frecuentemente asociados al TDAH; la presencia de un trastorno específico de la lectura puede tener un condicionante disfuncional específico en el apartado escolar [41]; la presencia de un trastorno oposicionista-desafiante generará como tal una repercusión clara en el componente social [42,43]. Por tanto, a la vista de la comorbilidad descrita, se muestra complejo cuantificar la disfunción propia del TDAH en estos casos. En este mismo sentido, debería valorarse cuidadosamente la necesidad de un abordaje específico del TDAH (farmacológico o no), por ejemplo, ante la presencia de un TDAH 'leve' con un trastorno específico de la lectura 'grave.'

\section{Evaluación de la disfunción del TDAH}

Indudablemente es necesaria una definición operativa del término 'disfunción', medidas válidas y objetivas para estimar dicha disfunción, y pautas claras para incorporar esta disfunción al diagnóstico del TDAH [34]. La falta de estimación de la repercusión marcada en el TDAH convierte este trastorno en un mero síndrome, un conjunto de síntomas que se correlacionan unos con otros; el término 'trastorno', en sí mismo, traduce una alteración funcional.

La definición de una interferencia en el funcionamiento personal del paciente [2] parece ambigua y tenue. En el sentido casi opuesto, por su concreción y gravedad, otros autores estiman situaciones como la repetición de curso, no finalizar la educación básica, rechazo social o pérdida de amigos, como una verdadera disfunción [35]. La primera definición parece ser más inherente al término 'síntoma' -observación subjetiva del individuo sobre una situación que se tipifica como anómala y disfuncional-; la segunda, puramente la consecuencia a medio o largo plazo.

Se muestra, por tanto, relevante intentar evaluar la disfunción de este trastorno [36]. Hasta la fecha, se han empleado diferentes medidas globales o multidimensionales. Entre las primeras, la más empleadas han sido la Children's Global Assessment Scale [44] y la Global Assessment of Functioning, esta última recomendada e incluida hasta el DSM-IV-TR [26]. Sin embargo, ambas escalas son globales y marcadamente contaminadas por el número de síntomas que presenta el paciente. Por otro lado, a pesar de los esfuerzos en establecer puntos de corte en el nú- mero de síntomas que el paciente debe presentar para un diagnóstico de TDAH, a nivel disfuncional no se establece ningún criterio claro para establecer 'la disfunción clínicamente significativa'. La recomendación de escalas multidimensionales llega al DSM-5, que sustituye la Global Assessment of Functioning por la recomendación de empleo de la World Health Organization Disability Assessment Schedule 2.0 [45]. Aunque carente de puntos de corte o valores de significancia clínica, el avance hacia una escala multidimensional parece importante. En contrapartida, es larga (36 ítems), inespecífica y todavía pobremente analizada-utilizada en el TDAH. Otras escalas multidimensionales han sido ocasionalmente empleadas en el TDAH [46-49]. De todas ellas, en los últimos años han mostrado más interés la NICHQ Vanderbilt Assessment Follow-Up [50], con un módulo específico de comportamiento y rendimiento, y particularmente la Weiss Functional Impairment Rating Scale (WFIRS) [51]. El módulo 'performance' de la primera recoge la situación escolar y social a través de ocho preguntas para padres y profesores, que son cuantificadas en una puntuación media de desempeño clínico, sin puntos de corte. La WFIRS recoge situaciones familiares, académicas, hábitos de vida, autoconcepto, adaptación social y comportamientos de riesgo a través de 50 preguntas; aunque es claramente más completa que la anterior, algunos ítems son propios de la propia sintomatología del TDAH o sus comorbilidades. El término 'repercusión' queda recogido con un punto de corte del propio test, y su elevada sensibilidad a los cambios con tratamiento ha favorecido su amplio empleo en los últimos años.

\section{Eficacia de los tratamientos}

La clara eficacia de los tratamientos farmacológicos sobre los síntomas nucleares del TDAH es incuestionable. Numerosos estudios demuestran una notable eficacia de los tratamientos psicoestimulantes y no psicoestimulantes, con tamaños de efecto realmente elevados [52-54]. En estudios a largo plazo, se ha podido constatar que los pacientes con TDAH muestran un peor pronóstico en diferentes esferas (funcionamiento social, desarrollo académico, autoestima...) que individuos sin este trastorno [55]; de igual manera, los pacientes con TDAH que han recibido tratamiento farmacológico muestran un mejor funcionamiento global que los que no lo han recibido [55]. Sin embargo, la mejora en algunos dominios funcionales o clínicos (conductas antisociales, ocupación laboral, conductas adictivas...) a me- 
dio y largo plazo no parece ser tan significativa [55]. Esta circunstancia podría deberse a una menor eficacia de los tratamientos farmacológicos sobre la comorbilidad del TDAH, la disfunción ejecutiva o la desregulación emocional, entre otros aspectos, circunstancias que condicionan el desempeño natural del paciente con TDAH.

Cuando se evalúa la eficacia de las intervenciones no farmacológicas (suplementación con ácidos grasos, neurofeedback, entrenamiento cognitivo, intervenciones conductuales) sobre los síntomas cardinales del TDAH, usando estimaciones ciegas, los tamaños de efecto son bajos, entre 0,02 y 0,29 [56]. No obstante, se debe señalar que existe cierta controversia acerca de los criterios de selección de estudios y el procedimiento llevado a cabo para realizar estos estudios $[57,58]$. Además, la eficacia de estas técnicas cuando se evalúan otros aspectos más funcionales relacionados con el TDAH (estilos educativos parentales, interacción padres-hijos, habilidades sociales, funcionamiento cognitivo...) se ha mostrado superior $[59,60]$. En todo caso, no cabe duda de la necesidad de realizar nuevos estudios con mayor rigor metodológico para estimar la eficacia de las intervenciones no farmacológicas, como el entrenamiento cognitivo o el neurofeedback.

La eficacia de las intervenciones no farmacológicas sobre la disfunción del paciente con TDAH se ha evaluado pobremente. En relación con las terapias cognitivo-conductuales o psicosociales, se han aportado efectos estadísticamente significativos sobre el desempeño del paciente, social, académico [61] o funcional, evaluado a través de escalas como la Global Assessment of Functioning o la WFIRS $[62,63]$. Hantson et al mostraron la eficacia de un campamento terapéutico de verano, de dos semanas de duración, con un total de seis horas de intervención diarias (entrenamiento de habilidades sociales, musicoterapia, terapia de juego, terapia a través del ejercicio y arte-terapia) sobre el funcionamiento global del paciente, evaluado a través de la WFIRS; los tamaños de efecto descritos en esta escala se situaban entre 0,7 y 1,5 , dependientes del dominio [64]. Es necesaria una mayor evidencia sobre la eficacia de estas medidas, con estimación ciega, para estimar su verdadera utilidad sobre el funcionamiento clínico de estos pacientes.

En el apartado farmacológico, la eficacia de los diferentes tratamientos sobre el desempeño funcional de los pacientes con TDAH ha cobrado un marcado interés en los últimos años. La WFIRS ha sido la más empleada en este sentido. Los tamaños de efecto de los tratamientos farmacológicos sobre la disfunción evaluada con esta escala siguen siendo moderada o marcadamente significativos [65-67]; sin embargo, esta eficacia parece menor que cuando se evalúa en relación con la mejora sobre los síntomas cardinales. Así, tamaños de efectos de 0,32, 0,76, 1,26 o 1,80 observados sobre la sintomatología del TDAH, tras tratamiento con atomoxetina, guanfacina retardada, metilfenidato de liberación osmótica o lisdexanfetamina, respectivamente, se reducen a 0,28, 0,44, 0,77 o 0,92, respectivamente, cuando se evalúa su incidencia sobre la WFIRS en el mismo estudio [68,69]. Esto podría traducir una menor sensibilidad de la WFIRS a los beneficios del tratamiento farmacológico, la presencia de síntomas más propios de la comorbilidad en esta escala o efectivamente una menor eficacia de los fármacos sobre el desempeño diario que sobre los síntomas característicos del TDAH. En contrapartida, estos estudios tienen un diseño de corta duración (entre siete y nueve semanas), por lo que parece lógico que, aunque la sintomatología puede mejorar inmediatamente, el funcionamiento evaluado en esta escala (situaciones familiares, académicas, hábitos de vida, autoconcepto, adaptación social y comportamientos de riesgo) podría mejorar posteriormente.

\section{Conclusiones}

A la vista de los datos expuestos, ante un trastorno tan heterogéneo clínicamente como el TDAH, sujeto sintomáticamente a la modulación por su elevada comorbilidad, parece trascendental la evaluación firme y objetiva de la disfunción. Los esfuerzos realizados en las últimas décadas en la tipificación sintomática deberán trasladarse a la tipificación disfuncional. Entender la disfunción (y, por tanto, la tipificación de 'trastorno') como una simple interferencia parece una banalización excesiva.

El empleo más sistemático de escalas para la evaluación de la disfunción en el TDAH podrá aportar un conocimiento más preciso de la afectación del trastorno sobre la vida del paciente a medio y largo plazo. Del mismo modo, podrá permitir una evaluación más ecológica de la eficacia de las intervenciones terapéuticas en estos tiempos.

\section{Bibliografía}

1. Willcutt EG. The prevalence of DSM-IV attention-deficit/ hyperactivity disorder: a meta-analytic review. Neurotherapeutics 2012; 9: 490-9.

2. American Psychiatric Association. Diagnostic and statistical manual of mental disorders, fifth edition (DSM-5). Washington DC: APA; 2013.

3. Organización Mundial de la Salud. CIE-10. Trastornos mentales y del comportamiento: descripciones clínicas y pautas para el diagnóstico. Madrid: Meditor; 1992. 
4. Sonuga-Barke EJ, Sergeant JA, Nigg J, Willcutt E. Executive dysfunction and delay aversion in attention deficit hyperactivity disorder: nosologic and diagnostic implications. Child Adolesc Psychiatr Clin N Am 2008; 17: 367-84.

5. Castellanos FX, Sonuga-Barke EJ, Milham MP, Tannock R. Characterizing cognition in ADHD: beyond executive dysfunction. Trends Cogn Sci 2006; 10: 117-23.

6. Roth RM, Saykin AJ. Executive dysfunction in attentiondeficit/hyperactivity disorder: cognitive and neuroimaging findings. Psychiatr Clin North Am 2004; 27: 83-96.

7. Albert J, López-Martín S, Fernández-Jaén A, Carretié L. Alteraciones emocionales en el trastorno por deficit de atencion/hiperactividad: datos existentes y cuestiones abiertas. Rev Neurol 2008; 47: 39-45.

8. López-Martín S, Albert J, Fernández-Jaén A, Carretié L. Emotional response inhibition in children with attentiondeficit/hyperactivity disorder: neural and behavioural data. Psychol Med 2015; Feb 24. [Epub ahead of print].

9. Childress AC, Sallee FR. Emotional lability in patients with attention-deficit/hyperactivity disorder: impact of pharmacotherapy. CNS Drugs 2015; 29: 683-93.

10. Zhang YF, Sun GX. A Meta analysis of family risk factors for attention deficit hyperactivity disorder. Zhongguo Dang Dai Er Ke Za Zhi 2015; 17: 721-5.

11. Pires TO, Da Silva CM, De Assis SG. Association between family environment and attention deficit hyperactivity disorder in children -mothers' and teachers' views. BMC Psychiatry 2013; 13: 215 .

12. Miranda A, Colomer C, Fernández MI, Presentación MJ, Roselló B. Analysis of personal and family factors in the persistence of attention deficit hyperactivity disorder: results of a prospective follow-up study in childhood. PLoS One 2015; 10: e0128325.

13. Du Prel Carroll X, Yi H, Liang Y, Pang K, Leeper-Woodford S, Riccardi P, et al. Family-environmental factors associated with attention deficit hyperactivity disorder in Chinese children: a case-control study. PLoS One 2012; 7: e50543.

14. Mohr-Jensen C, Steen-Jensen T, Bang-Schnack M, Thingvad H. What do primary and secondary school teachers know about ADHD in children? Findings from a systematic review and a representative, nationwide sample of Danish teachers. J Atten Disord 2015; Aug 22. [Epub ahead of print].

15. Aguiar AP, Kieling RR, Costa AC, Chardosim N, Dorneles BV, Almeida MR, et al. Increasing teachers' knowledge about ADHD and learning disorders: an investigation on the role of a psychoeducational intervention. J Atten Disord 2014; 18: 691-8.

16. Pfiffner LJ, Yee Mikami A, Huang-Pollock C, Easterlin B, Zalecki C, McBurnett $\mathrm{K}$. A randomized, controlled trial of integrated home-school behavioral treatment for ADHD, predominantly inattentive type. J Am Acad Child Adolesc Psychiatry 2007; 46: 1041-50.

17. Ostberg M, Rydell AM. An efficacy study of a combined parent and teacher management training programme for children with ADHD. Nord J Psychiatry 2012; 66: 123-30.

18. Fundació Sant Joan de Déu, coord; Grupo de Trabajo de la Guía de Práctica Clínica sobre el Trastorno por Déficit de Atención con Hiperactividad (TDAH) en Niños y Adolescentes. Guía de práctica clínica sobre el trastorno por déficit de atención con hiperactividad (TDAH) en niños y adolescentes. Madrid: Ministerio de Ciencia e Innovación; 2010. URL: http: // www.guiasalud.es/GPC/GPC_477_TDAH_AIAQS_compl.pdf

19. Kendall T, Taylor E, Perez A, Taylor C; Guideline Development Group. Diagnosis and management of attention-deficit/ hyperactivity disorder in children, young people, and adults: summary of NICE guidance. BMJ 2008; 337: a1239.

20. Pliszka S, AACAP Work Group on Quality Issues. Practice parameter for the assessment and treatment of children and adolescents with attention-deficit/hyperactivity disorder. J Am Acad Child Adolesc Psychiatry 2007; 46: 894-921.

21. Subcommittee on Attention-Deficit/Hyperactivity Disorder Steering Committee on Quality Improvement Management, Wolraich M, Brown L, Brown RT, DuPaul G, Earls M, et al. ADHD: clinical practice guideline for the diagnosis, evaluation, and treatment of attention-deficit/hyperactivity disorder in children and adolescents. Pediatrics 2011; 128: 1007-22.

22. Del Barrio V. Raíces y evolución del DSM. Rev Hist Psicol 2009; 30: 81-90.

23. Lange KW, Reichl S, Lange KM, Tucha L, Tucha O. The history of attention deficit hyperactivity disorder. Atten Defic Hyperact Disord 2010; 2: 241-55.

24. American Psychiatric Association. Guía de consulta de los criterios diagnósticos del DSM-5. Madrid: Editorial Médica Panamericana; 2014.

25. American Psychiatric Association. Diagnostic and statistical manual of mental disorders: diagnostic criteria from DSM-IV. 2 ed. Washington DC: APA; 1994.

26. American Psychiatric Association. Diagnostic and statistical manual of mental disorders, fourth edition, text revised (DSM-IV-TR). Arlington, VA: APA; 2005.

27. Cook J, Knight E, Hume I, Qureshi A. The self-esteem of adults diagnosed with attention-deficit/hyperactivity disorder (ADHD): a systematic review of the literature. Atten Defic Hyperact Disord 2014; 6: 249-68.

28. Edbom T, Lichtenstein P, Granlund M, Larsson JO. Long-term relationships between symptoms of attention deficit hyperactivity disorder and self-esteem in a prospective longitudinal study of twins. Acta Paediatr 2006; 95: 650-7.

29. Danckaerts M, Sonuga-Barke EJ, Banaschewski T, Buitelaar J, Dopfner M, Hollis C, et al. The quality of life of children with attention deficit/hyperactivity disorder: a systematic review. Eur Child Adolesc Psychiatry 2010; 19: 83-105.

30. Agarwal R, Goldenberg M, Perry R, Ishak WW. The quality of life of adults with attention deficit hyperactivity disorder: a systematic review. Innov Clin Neurosci 2012; 9: 10-21.

31. Brook U, Boaz M. Adolescents with attention deficit and hyperactivity disorder/learning disability and their proneness to accidents. Indian J Pediatr 2006; 73: 299-303.

32. Swensen A, Birnbaum HG, Ben Hamadi R, Greenberg P, Cremieux PY, Secnik K. Incidence and costs of accidents among attention-deficit/hyperactivity disorder patients. J Adolesc Health 2004; 35: 346 e1-9.

33. Gordon M, Antshel K, Faraone S, Barkley R, Lewandowski L, Hudziak JJ, et al. Symptoms versus impairment: the case for respecting DSM-IV's criterion D. J Atten Disord 2006; 9: 465-75.

34. Gathje RA, Lewandowski LJ, Gordon M. The role of impairment in the diagnosis of ADHD. J Atten Disord 2008; 11: 529-37.

35. Barkley R, Cunningham C, Gordon M, Faraone S, Lewandowski L, Murphy KR. ADHD symptoms vs. impairment: revisited. ADHD Report 2006; 14: 1-9.

36. Hodges K, Gust J. Measures of impairment for children and adolescents. J Ment Health Adm 1995; 22: 403-13.

37. Catalá-López F, Peiró S, Ridao M, Sanfélix-Gimeno G, Génova-Maleras R, Catalá MA. Prevalence of attention deficit hyperactivity disorder among children and adolescents in Spain: a systematic review and meta-analysis of epidemiological studies. BMC Psychiatry 2012; 12: 168

38. Gillberg C, Gillberg IC, Rasmussen P, Kadesjo B, Soderstrom H, Rastam M, et al. Co-existing disorders in ADHD -implications for diagnosis and intervention. Eur Child Adolesc Psychiatry 2004; 13 (Suppl 1): S80-92.

39. Steinhausen HC, Novik TS, Baldursson G, Curatolo P, Lorenzo MJ, Rodrigues Pereira R, et al. Co-existing psychiatric problems in ADHD in the ADORE cohort. Eur Child Adolesc Psychiatry 2006; 15 (Suppl 1): S25-9.

40. Hodgkins P, Setyawan J, Mitra D, Davis K, Quintero J, Fridman M, et al. Management of ADHD in children across Europe: patient demographics, physician characteristics and treatment patterns. Eur J Pediatr 2013; 172: 895-906.

41. Shaywitz SE, Shaywitz BA. Dyslexia (specific reading disability). Biol Psychiatry 2005; 57: 1301-9.

42. Burke JD, Loeber R, Birmaher B. Oppositional defiant disorder and conduct disorder: a review of the past 10 years, part II. J Am Acad Child Adolesc Psychiatry 2002; 41: 1275-93.

43. Loeber R, Burke JD, Lahey BB, Winters A, Zera M. Oppositional defiant and conduct disorder: a review of the past 10 years, part I. J Am Acad Child Adolesc Psychiatry 2000; 39: 1468-84. 
44. Shaffer D, Gould MS, Brasic J, Ambrosini P, Fisher P, Bird H, et al. A Children's Global Assessment Scale (CGAS). Arch Gen Psychiatry 1983; 40: 1228-31.

45. Gold LH. DSM-5 and the assessment of functioning: the World Health Organization Disability Assessment Schedule 2.0 (WHODAS 2.0). J Am Acad Psychiatry Law 2014; 42: 173-81.

46. Mugnaini D, Fabiano GA. The evaluation of impairment in the attention deficit/hyperactivity disorder: the contribution of the impairment rating scale. Minerva Pediatr 2006; 58: 159-66.

47. Rotger S, Richarte V, Nogueira M, Corrales M, Bosch R, Vidal R, et al. Functioning Assessment Short Test (FAST): validity and reliability in adults with attention-deficit/ hyperactivity disorder. Eur Arch Psychiatry Clin Neurosci 2014; 264: 719-27.

48. Park JH, Lee SI, Schachar RJ. Reliability and validity of the child and adolescent functioning impairment scale in children with attention-deficit/hyperactivity disorder. Psychiatry Investig 2011; 8: 113-22.

49. Gajria K, Kosinski M, Sikirica V, Huss M, Livote E, Reilly K, et al. Psychometric validation of the Weiss Functional Impairment Rating Scale-Parent Report Form in children and adolescents with attention-deficit/hyperactivity disorder Health Qual Life Outcomes 2015; 13: 184

50. American Academy of Pediatrics and National Initiative for Children's Healthcare Quality. NICHQ Vanderbilt Assessment Scales. Boston: NICHQ; 2002.

51. Epstein JN, Weiss MD. Assessing treatment outcomes in attention-deficit/hyperactivity disorder: a narrative review. Prim Care Companion CNS Disord 2012; 14: doi:10.4088/ PCC.11r0133.

52. Faraone SV, Glatt SJ. A comparison of the efficacy of medications for adult attention-deficit/hyperactivity disorder using metaanalysis of effect sizes. J Clin Psychiatry 2010; 71: 754-63.

53. Faraone SV, Buitelaar J. Comparing the efficacy of stimulants for ADHD in children and adolescents using meta-analysis. Eur Child Adolesc Psychiatry 2010; 19: 353-64.

54. Stuhec M, Munda B, Svab V, Locatelli I. Comparative efficacy and acceptability of atomoxetine, lisdexamfetamine, bupropion and methylphenidate in treatment of attention deficit hyper activity disorder in children and adolescents: a meta-analysis with focus on bupropion. J Affect Disord 2015; 178: 149-59.

55. Shaw M, Hodgkins P, Caci H, Young S, Kahle J, Woods AG, et al. A systematic review and analysis of long-term outcomes in attention deficit hyperactivity disorder: effects of treatment and non-treatment. BMC Med 2012; 10: 99.

56. Sonuga-Barke EJ, Brandeis D, Cortese S, Daley D, Ferrin M, Holtmann M, et al. Nonpharmacological interventions for ADHD: systematic review and meta-analyses of randomized controlled trials of dietary and psychological treatments. Am J Psychiatry 2013; 170: 275-89.

57. Chronis-Tuscano A, Chacko A, Barkley R. Key issues relevant to the efficacy of behavioral treatment for ADHD. Am J Psychiatry 2013; 170: 799.
58. Arns M, Strehl U. Evidence for efficacy of neurofeedback in ADHD? Am J Psychiatry 2013; 170: 799-800.

59. Daley D, Van der Oord S, Ferrin M, Danckaerts M, Doepfner M Cortese S, et al. Behavioral interventions in attention-deficit/ hyperactivity disorder: a meta-analysis of randomized controlled trials across multiple outcome domains. J Am Acad Child Adolesc Psychiatry 2014; 53: 835-47.

60. Hodgson K, Hutchinson AD, Denson L. Nonpharmacological treatments for ADHD: a meta-analytic review. J Atten Disord 2014; 18: 275-82.

61. Fabiano GA, Schatz NK, Aloe AM, Chacko A, ChronisTuscano A. A systematic review of meta-analyses of psychosocial treatment for attention-deficit/hyperactivity disorder. Clin Child Fam Psychol Rev 2015; 18: 77-97.

62. Knouse LE, Sprich S, Cooper-Vince C, Safren SA. Attentiondeficit/hyperactivity disorder symptom profiles in medicationtreated adults entering a psychosocial treatment program. J ADHD Relat Disord 2009; 1: 34-48.

63. Vidal R, Castells J, Richarte V, Palomar G, García M, Nicolau R, et al. Group therapy for adolescents with attention-deficit/ hyperactivity disorder: a randomized controlled trial. J Am Acad Child Adolesc Psychiatry 2015; 54: 275-82.

64. Hantson J, Wang PP, Grizenko-Vida M, Ter-Stepanian M, Harvey W, Joober R, et al. Effectiveness of a therapeutic summer camp for children with ADHD: phase I clinical intervention trial. J Atten Disord 2012; 16: 610-7.

65. Nagy P, Hage A, Coghill DR, Caballero B, Adeyi B, Anderson CS et al. Functional outcomes from a head-to-head, randomized, double-blind trial of lisdexamfetamine dimesylate and atomoxetine in children and adolescents with attentiondeficit/hyperactivity disorder and an inadequate response to methylphenidate. Eur Child Adolesc Psychiatry 2016; 25: 141-9.

66. Banaschewski T, Johnson M, Lecendreux M, Zuddas A, Adeyi B, Hodgkins P, et al. Health-related quality of life and functional outcomes from a randomized-withdrawal study of long-term lisdexamfetamine dimesylate treatment in children and adolescents with attention-deficit/hyperactivity disorder. CNS Drugs 2014; 28: 1191-203.

67. Stein MA, Sikirica V, Weiss MD, Robertson B, Lyne A, Newcorn JH. Does guanfacine extended release impact functional impairment in children with attention-deficit/ hyperactivity disorder? Results from a randomized controlled trial. CNS Drugs 2015; 29: 953-62.

68. Hervas A, Huss M, Johnson M, McNicholas F, Van Stralen J, Sreckovic S, et al. Efficacy and safety of extended-release guanfacine hydrochloride in children and adolescents with attention-deficit/hyperactivity disorder: a randomized, controlled, phase III trial. Eur Neuropsychopharmacol 2014; 24: 1861-72.

69. Banaschewski T, Soutullo C, Lecendreux M, Johnson M, Zuddas A, Hodgkins P, et al. Health-related quality of life and functional outcomes from a randomized, controlled study of lisdexamfetamine dimesylate in children and adolescents with ADHD. CNS Drugs 2013; 27: 829-40.

\section{Dysfunction in attention deficit hyperactivity disorder: assessment and response to treatment}

Summary. Attention deficit hyperactivity disorder (ADHD) is a heterogeneous, symptomatically complex disorder. Its cardinal symptom, the presence of dysexecutive problems, emotional dysregulation of many of them and its own comorbidity, among others, will condition its clinical expression and the dysfunction. Classifying ADHD as a 'disorder' calls for an accurate assessment of the terms 'dysfunction' or 'repercussion'. The progress made in the classification and quantification of the symptoms characterising ADHD should be applied to measuring and objectifying dysfunction. Considering dysfunction as a simple interference, however clear it may be, could lead to an overestimation of the diagnosis of this disorder. Just as its estimation is essential for a diagnosis, it is also necessary for the correct evaluation of the efficacy of the therapeutic interventions, especially in the medium and long term. Further studies are needed in this sense to appraise the efficacy of the treatments, whether pharmacological or not, in different domains (social relationship, learning, self-esteem, quality of life, accidents, etc.).

Key words. ADHD. Attention deficit. DSM-5. Dysfunction. Repercussion. WFIRS. 